HEAD AND NECK

\title{
VITOM-3D assisted neck dissection via a retroauricular approach (RAND-3D): a preclinical investigation in a cadaver lab
}

\author{
Dissezione laterocervicale mediante approccio retroauricolare assistito da sistema \\ VITOM-3D (RAND-3D): studio preclinico in cadaver lab \\ Erika Crosetti ${ }^{1}$, Giulia Arrigoni ${ }^{1}$, Andrea Manca ${ }^{1}$, Marco Fantini ${ }^{1}$, Alessandra Caracciolo ${ }^{1}$, Francesco Sardanapoli, \\ Giovanni Succo ${ }^{1,2}$ \\ ${ }^{1}$ Head and Neck Oncology Unit, Candiolo Cancer Institute, FPO - IRCCS, Candiolo (TO), Italy; ${ }^{2}$ Department of Oncology, University \\ of Turin, Orbassano (TO), Italy
}

\begin{abstract}
SUMMARY
Objective. The recent introduction of 3D exoscopic surgery has allowed interesting technical improvements in head and neck surgery resulting in technical solutions that are also applicable to neck dissection. The aim is to replace robotic surgery while minimising the costs of the procedure.

Methods. Based on these considerations, we conducted a preclinical investigation in the cadaver lab focused on approaching conventional neck dissection using a retroauricular incision, and evalute the applications and usefulness of the Storz 3D Exoscopic System at different stages of the surgical procedure. The acronym RAND-3D (3D exoscopic surgery) was coined to describe the application of this optical tool in neck dissection.

Results. The current study in the cadaver lab indicates that RAND-3D is an acceptable alternative operating technique in performing neck dissection by a retroauricular approach. Technically feasible and safe, this technique assures a complete compartment-oriented dissection without damaging major vascular or nervous structures.

Conclusions. This approach can be used in selected cases with a clear cosmetic benefit and represents a valid alternative to endoscopic- and robotic-assisted neck dissection.
\end{abstract}

KEY WORDS: 3D, exoscope, neck dissection, 3D surgery

\section{RIASSUNTO}

Obiettivo. Il recente avvento della chirurgia esoscopica $3 D$ ha consentito, nell'ambito della chirurgia cervico-cefalica, l'introduzione di interessanti innovazioni tecnologiche, risultanti in soluzioni tecniche applicabili anche alla chirurgia del collo, con l'obiettivo di rappresentare un'alternativa alla chirurgia robotica, minimizzando i costi della procedura. Metodi. Sulla base di queste considerazioni abbiamo recentemente condotto uno studio pre-clinico nel nostro cadaver lab, focalizzato sull'esecuzione dello svuotamento linfonodale laterocervicale con approccio retroauricolare, valutando le applicazioni e l'utilità del Sistema Esoscopico 3D Storz nei vari steps dell'intervento. Abbiamo coniato l'acronimo di RAND-3D (chirurgia esoscopica $3 D$ ) per descrivere l'impiego di questo tool ottico nell'esecuzione di tale procedura chirurgica.

Risultati. Il seguente studio condotto nel cadaver lab ha indicato che la tecnica RAND-3D rappresenta una valida alternativa per l'esecuzione dello svuotamento del collo con approccio retroauricolare. Questa metodica, tecnicamente fattibile e sicura, permette una dissezione compartimentale, senza danno alle principali strutture vascolo-nervose cervicali. Conclusioni. Questo approccio può essere impiegato in casi selezionati con un chiaro finalismo estetico, rappresentando una valida alternativa alle metodiche endoscopiche e robotiche.

PAROLE CHIAVE: 3D, esoscopio, svuotamento laterocervicale, chirurgia 3D
Received: March 22, 2020

Accepted: August 6, 2020

\section{Correspondence}

Erika Crosetti

Head and Neck Oncology Unit, FPO IRCCS, Candiolo Cancer Institute, 10060 Candiolo (Turin) Italy Tel. + 390119933663

E-mail: erika.crosetti@ircc.it

Funding

This research was funded by: Regione Piemonte AD FUNCTIONEM (years 2015-2018); FPRC 5xmille 2016 Ministero della Salute Progetto ARDITE; Fondi Ricerca Corrente 2020, Ministero della Salute.

Conflict of interest

The Authors declare no conflict of interest.

How to cite this article: Crosetti E, Arrigoni G, Manca A, et al. VITOM-3D assisted neck dissection via a retroauricular approach (RAND3D): a preclinical investigation in a cadaver lab. Acta Otorhinolaryngol Ital 2020;40:343-351. https://doi.org/10.14639/0392-100X-N0757

(C) Società Italiana di Otorinolaringoiatria e Chirurgia Cervico-Facciale

\section{(c) (1) $(9)$}

This is an open access article distributed in accordance with the CC-BY-NC-ND (Creative Commons Attribution-NonCommercial-NoDerivatives 4.0 International) license. The article can be used by giving appropriate credit and mentioning the license, but only for non-commercial purposes and only in the original version. For further information: https:// creativecommons.org/licenses/by-nc-nd/4.0/deed.en 


\section{Introduction}

Traditionally, surgery for head and neck malignancies involves open neck approaches aimed at radical removal of the primary tumour together with cervical lymph nodes, allowing the surgeon to obtain good control of the disease; however, occasionally, negative post-operative aesthetic and functional sequelae result.

The need to implement surgical approaches that minimise the morbidity and impact of treatments has given great impetus to the development of minimally invasive techniques, such as laser surgery and robotic surgery ${ }^{1,2}$.

As far as treatment of the neck is concerned, to date, not much has changed in terms of surgical technique, since the cervical open neck approach remains the conventional one, even in the case of transoral surgery on the primary tumour, and also resulting in the paradox of minimally invasive surgery on $\mathrm{T}$ and conventional surgery on $\mathrm{N}$, and weakening the concept of minimal invasiveness. This has prompted surgeons to try to develop minimally invasive neck dissection techniques using endoscopic and robotic approaches. Endoscopic-assisted and robot-assisted neck dissection present limitations and disadvantages. Of these, the main the duration of surgery, longer than the standard approach, the need for surgeons to develop skills in endoscopic and robotic techniques and the overall higher costs of the procedures (about six times higher in the Korean experience using robotic neck dissection) ${ }^{3-7}$.

The recent introduction of 3D exoscopic surgery has allowed interesting technical improvements in head and neck surgery resulting in technical solutions that are also applicable to neck dissection, with the aim of replacing robotic surgery but minimising the costs of the procedure ${ }^{8}$.

Specifically, exoscopic surgery is based on the use of a sterile 3D optical device, called an exoscope, placed in the surgical field and oriented with different inclinations but technically about $40 \mathrm{~cm}$ distant from it. The exoscope also illuminates the surgical field in depth, since it is equipped with two light cables with Xenon sources, and produces $4 \mathrm{~K}$ images on a 55" screen positioned about 3-4 metres from the surgeon. In some steps in the surgical procedure, the exoscope replaces the surgeon's eyes thereby improving magnification. This allows the possibility to carry out the neck dissection through a retroauricular incision, combining direct and 3D exoscopic vision and resulting in operating times no longer than $20 \%$ of previous times and with a comparable level of feasibility, determined by the perfect visualisation of the surgical field even in the more remote cervical areas (levels Ib-IV).

The goal of this study is to analyse the technical details of neck dissection in a cadaver lab carried out using a remote approach in what is virtually conventional surgery but enhanced using 3D exoscopic technology in more remote areas.

The primary end point was to demonstrate the effectiveness of this technology in retroauricular or facelift approaches to neck dissection. The secondary end point was to assess which node levels could be dissected using the 3D exoscopic system, the docking and setting of the procedure, and the advantages and limitations of the technique. The tertiary end point was to become familiar with a traditional technique carried out using a remote approach, to be considered as a rescue procedure in the case of major bleeding complications during a robotic neck dissection.

\section{Materials and methods}

Four human cadavers were obtained from the Italian Academy of Anatomical Dissection (AIAD). Three cadavers were male and one was female. Dissection was carried out bilaterally on each cadaver by two senior head and neck surgeons.

\section{Technology}

The video telescope operating microscope (VITOM ${ }^{\circledR}$; Karl Storz, Tuttlingen, Germany) system is an exoscope with $0^{\circ}$ or $90^{\circ}$ camera options. VITOM-3D has zoom and focus functions, integrated illumination and horizontal alignment. The camera offers a magnification of $1-2 \times$ depending on the working distance and the size and resolution of the monitor used. The surgical field is visualised by a camera system which supports an output signal of $1920 \times 1080 p$ and with a 16:9 image format; progressive scanning delivers crystal-clear images with smooth motion, even if there is rapid movement. The 3-chip sensor technology in the camera head ensures natural colour rendition.

The exoscope is positioned on a versatile self-supporting arm, specially developed for use with the VITOM $^{\circledR}$ system, which allows a simple and precise positioning of the system. The arm is mounted on a mobile trolley and can be transported and positioned quickly in the operating room. Thanks to its pneumatic cushioning, the arm allows weight compensation for the VITOM ${ }^{\circledR}$ system. The braking effect of each individual joint can be set individually. Furthermore, the arm is noted for its easy handling: the VITOM ${ }^{\circledR}$ system can be easily positioned with one hand. Different directions of view are possible depending on the surgical site being treated.

HD imaging also provides a sense of depth to improve anatomical orientation. The camera is connected to a 3D monitor and 3D passive-polarised glasses, with an antifog coating, or 3D clip-on glasses, circularly polarised, are worn to view the monitor. An intuitive control unit with 3D wheel (joystick) with four programmable function keys is 
used to control the camera. The joystick can be used with the sterile coating by the surgical assistants, or without the coating by other members of the surgical team not directly working on the operating field. The joystick can also be used to control an instrument holding system. To increase the illumination of the operating field, it is possible to place an annular illuminator device on the head of the exoscope, connected to a second Xenon light source.

In order to carry out a hybrid procedure in which a large part of the operation is performed under direct control and only the dissection of the most distant layers from the incision (levels IV and $\mathrm{Ib}$ ) is performed under exoscopic 3D vision, the polarising lenses were adapted to the $3.5 \times$ magnification operating loupes.

\section{Operating lab setting}

The first and second surgeons are seated on the same side as the neck dissection. The 55" monitor is positioned contralaterally in front of them, at a distance of about 2.5 metres. The assistant is placed at the head of the cadaver. The endoscopic cart is positioned at the bottom of the operating bed with a second 3D monitor for the assistant. The first and second surgeons also wear operating loupes over polarising clip-on lenses to view the monitor when necessary. At present, the exoscope is mounted on a mechanical holder, positioned behind the two surgeons and oriented towards the level to be dissected and is moved by an assistant. The latter controls the exoscope joystick. All team members wear 3D glasses. Throughout the operation, the exoscope is positioned behind the first two operators and directed towards the level to be dissected (Fig. 1).

\section{Results}

The cadaver is placed in the supine passive position with neck slightly extended and turned contralateral to the side that will be operated. It is useful to identify and mark the external jugular vein on the skin, if present, and the margins of the platysma/sternocleidomastoid muscle.

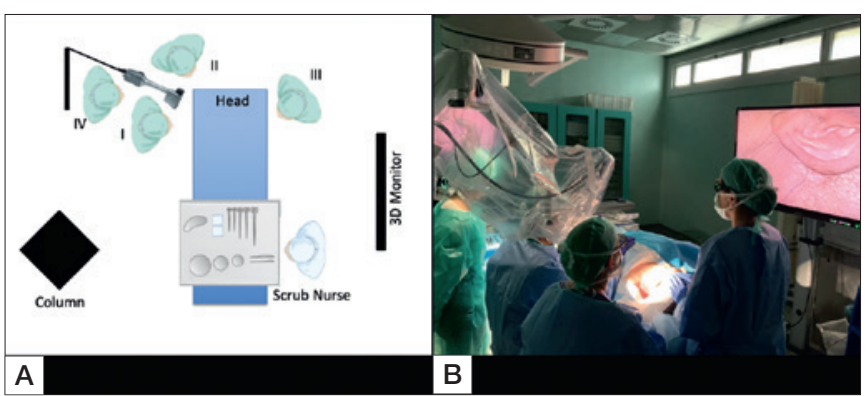

Figure 1. A) Schematic of operating lab setting. B) Operating lab setting.
A modified retroauricular or facelift skin incision is performed without pre-auricular extension (Fig. 2). The modified facelift incision, given its extension into the pre-auricular region, allows for more straightforward and faster dissection, providing a wider 'working space', especially for patients who need a dissection of level $\mathrm{Ib}$. The median operating time for cutaneous flap harvesting and placement of skin retractor was 38 minutes (range 25-78 minutes). The median operating time for 3D exoscopic-assisted neck dissection (levels $\mathrm{Ib}-\mathrm{V}$ ) was 83.2 minutes (range 73-103 minutes).

Neck dissection was successfully performed from levels I to $\mathrm{V}$ in all four cadavers. The skin flap is elevated under direct vision along a subplatysmal plane, and the inferior aspect of the parotid gland, the great auricular nerve, and the external jugular vein is identified which could be ligated and sectioned as well as spared. The latter two anatomical structures represent the anatomical landmarks used to identify the correct subplatysmal plane.

The anterolateral margin of the sternocleidomastoid muscle and the angle of the mandible are then identified. The angle of the mandible, an important landmark for the mandibular branch of the VII ${ }^{\text {th }}$ cranial nerve, is then marked using a sterile pencil, over the fat tissue so as to always maintain these anatomical structures in the field of view. The dissection proceeds with the aid of the 3D exoscope. We identified inferiorly the upper margin of the clavicle, medially the anterior belly of the contralateral digastric muscle, and laterally the anteromedial margin of the trape-

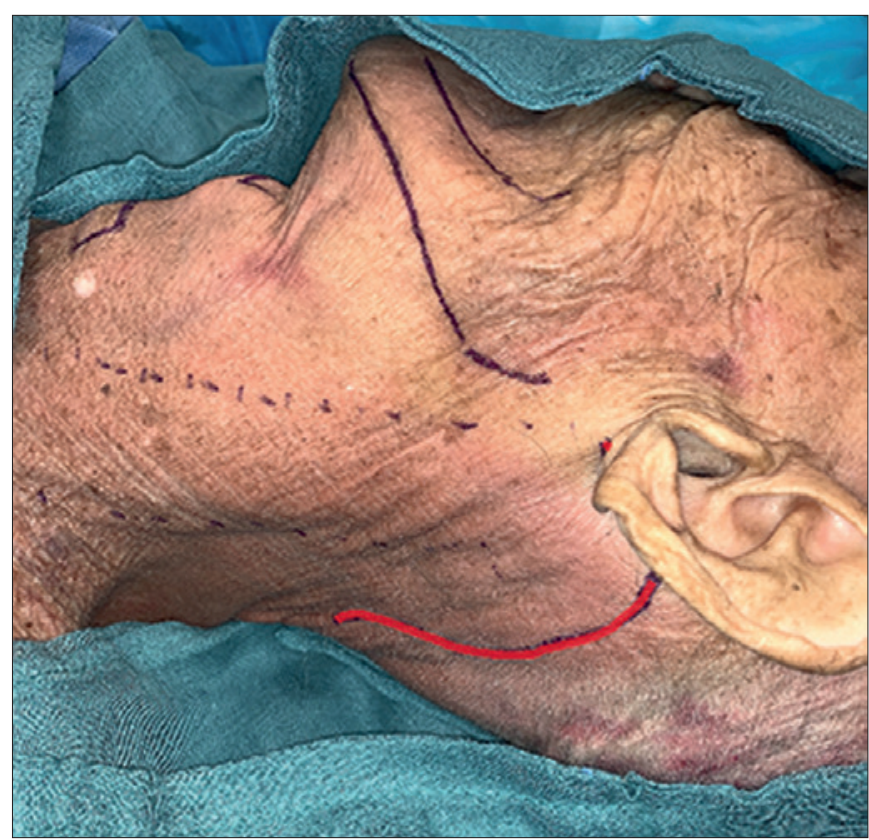

Figure 2. Modified retroauricular or facelift skin incision (red line). 


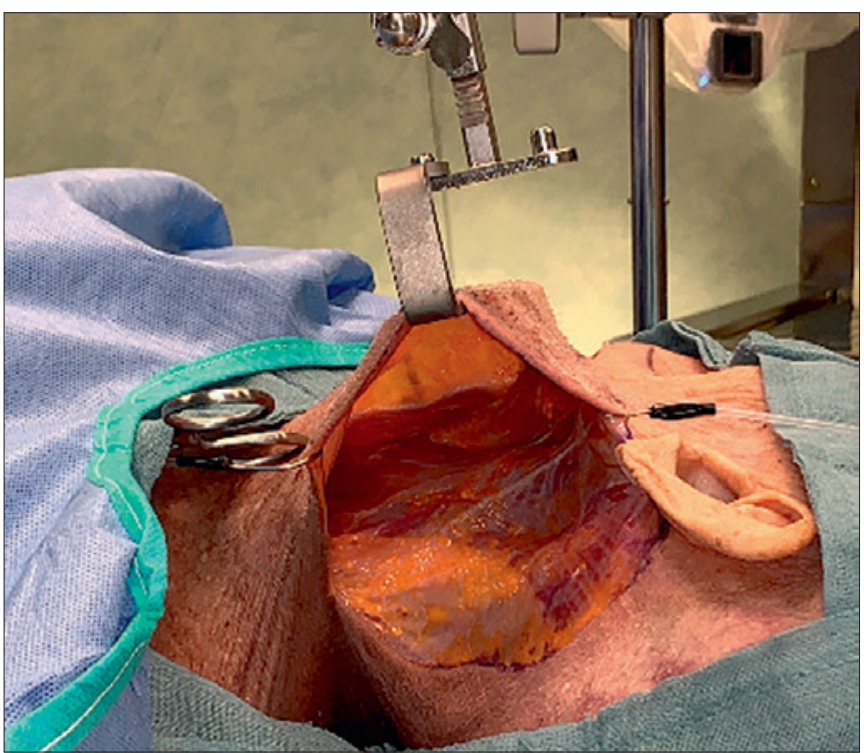

Figure 3. Surgical view (left side) after self-retaining retractor placement.

zius muscle. A self-retaining Koh's retractor or Mikulitz's spatula is then placed and kept raised by the third assistant (Fig. 3). In this step, the first and second surgeons work next to each other. To better harvest the subplatysmal flap up to the clavicle inferiorly, it is preferable that the first surgeon is positioned at the head of the cadaver or that this step is carried out by the second surgeon.

Different levels in the neck (I, IIa, IIb, III, IV, Va, Vb) are then dissected as required using the traditional surgical technique, with vascular clips and haemostatic cutting/ coagulation devices such as bipolar scissors and LigaSure Maryland forceps. Surgical instruments (forceps, scissors) must be a minimum length of $24 \mathrm{~cm}$ so as to be able to work in deep spaces without any difficulty.

The aid provided by the 3D vision consists of useful lighting of more distant areas of the neck and improving the visualisation of anatomic structures. The visualisation is exactly the same for both surgeons providing great precision in the surgical manoeuvers.

At the end of the procedure, we decided not to send the specimen to the pathologist because the main aim of our preclinical investigation was evaluation of the feasibility of the approach.

\section{Dissection of level II}

To dissect this level, the first surgeon is positioned on the same side as the neck dissection while the second surgeon stands next to the first surgeon towards the patient's head. The dissection proceeds in a medial $\rightarrow$ lateral direction quickly carried out under direct vision and only some hae-

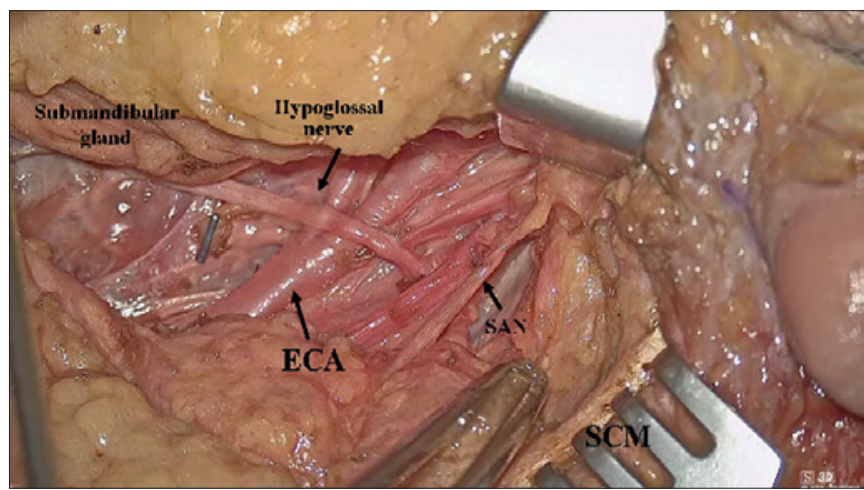

Figure 4. Surgical view (left side) after level lla dissection (ECA: external carotid artery; SAN: spinal accessory nerve; SCM: sternocleidomastoid muscle).

mostatic manoeuvers, carried out by the second surgeon around the hypoglossal nerve and thyrolinguofacial trunk, can benefit from exoscopic vision (Fig. 4). The neck dissection then proceeds with dissection of level IIb. The digastric muscle is retracted upwards by the third assistant using an Army-Navy retractor and the spinal accessory nerve (SAN) is dissected (Fig. 5). Level IIb is completely dissected under direct vision.

The percentage of surgery carried out under direct vision vs 3D exoscopic vision was calculated $(90 \%$ vs $10 \%)$. VITOM-3D was useful to the assistant to aid precision in haemostatic manoeuvers.

\section{Dissection of level III}

For dissection of level III, the operation always proceeds in a medial $\rightarrow$ lateral direction. Dissection from the deep plane and the medial-lateral shifting of the loose and fascial cellular tissue isolated so far allow the SAN and the cervical plexus to be identified. The specimen is then gently pulled downwards, and the perivasal fasciae cut with scissors (Fig. 6).

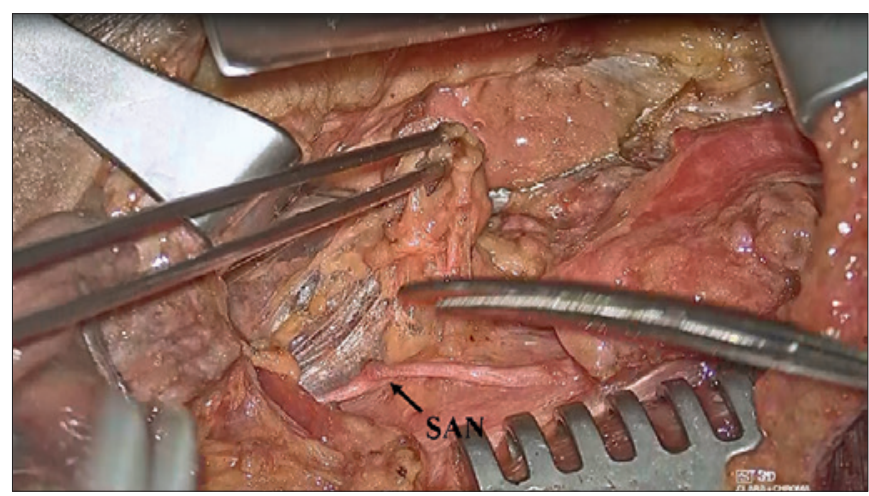

Figure 5. Surgical view (right side): the digastric muscle is retracted upwards by the third assistant using an Army-Navy retractor and the spinal accessory nerve (SAN) is dissected. 


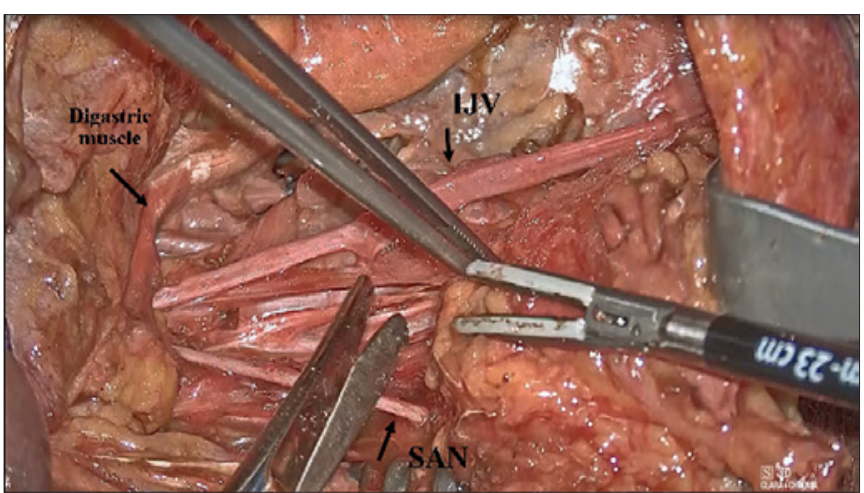

Figure 6. Surgical view (right side): for the dissection of level III, the operation always proceeds in a medial $\rightarrow$ lateral direction, from the deep plane and the medial-lateral shifting to allow the SAN and the cervical plexus to be identified. (IJV: internal jugular vein; SAN: spinal accessory nerve).

The positions of the surgeons and the exoscope are the same as in level II dissection. To dissect this level, the percentage of 3D exoscopic vision rose to $20 \%$, in particular when dissecting the lower part of this level, meaning that even the first surgeon can improve his accuracy.

\section{Dissection of levels IV-V}

For the dissection of levels IV and V, the first surgeon moves to the head of the cadaver. Another possibility is that the second surgeon proceeds with cervical dissection in this step. The dissection of level $\mathrm{V}$ is then carried out in a lateral $\rightarrow$ medial direction under direct vision in conventional mode without any particular difficulties. To dissect level IV, the retractor blade raising the skin flap is positioned towards the sternal notch. An assistant retracts the sternocleidomastoid muscle apart using a Richardson's retractor. The surgical instruments are directed caudally and the exoscope is directed towards the clavicle and sternal notch (Fig. 7).

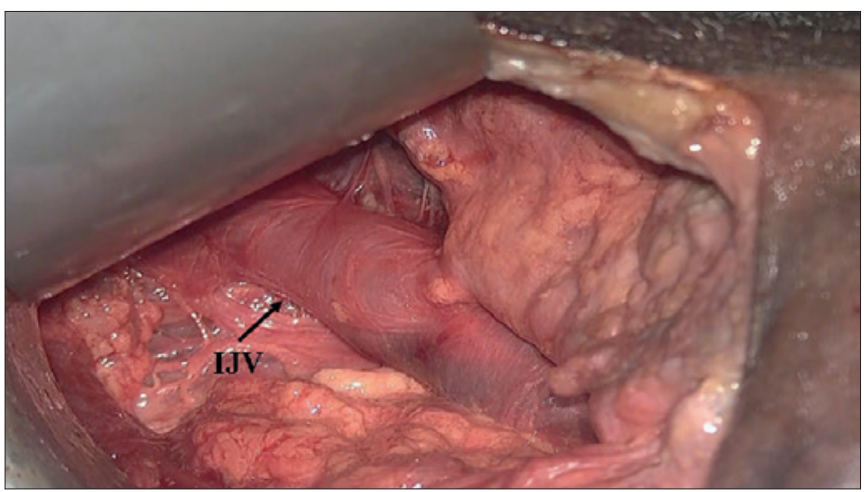

Figure 7. Surgical view (right side): dissection of level IV (the omohyoid muscle is retracted) (IJV: internal jugular vein).
When dissecting the lower part of level IV, to prevent any possible lymphorrhage due to damage to the thoracic duct/ great lymphatic vein, it is mandatory to place multiple vascular clips before cutting the fat tissue. The side of dissection did not have any significant effect on the performance of neck dissection.

This is undoubtedly the most challenging step in the operation because, at level IV, the working space is extremely narrow and the risk of an inadvertent injury to the internal jugular vein (IJV) or thoracic duct is high. Also in this step, the exoscope is useful for sharing the same good vision among all surgeons; however, the long working distance makes this dissection lengthy and difficult to manage, even for expert surgeons.

To facilitate dissection of level IV, and to dramatically reduce the risks of vascular/lymphatic injury, it is extremely useful to make a small incision $(5 \mathrm{~cm})$ midway between the cricoid cartilage and sternal notch, as is currently performed in thyroid surgery. The breadth of this incision should exceed the external border of the sternocleidomastoid muscle by $1 \mathrm{~cm}$. The concept is to create a service skin window, allowing the lateral margin of the sternocleidomastoid muscle, the upper belly of the omohyoid muscle, and the cervical vascular-nervous axis to be identified and then dissecting this level upward, reaching level III (Fig. 8). Highlighting the phrenic nerve and the brachial plexus, dissection of the inferior part of levels IV and V is completed, certainly the most delicate and difficult to manage step coming from a remote behind-the-ear approach.

This surgical solution is also useful when performing thyroid surgery for malignancies, where neck dissection is also required due to cervical metastases, allowing an effective surgical procedure and leaving only a small visible scar.

To dissect these levels, the rate of $3 \mathrm{D}$ exoscopic vision is about $20 \%$ when proceeding only via a retroauricular approach; while it is less than 5\% when proceeding via the

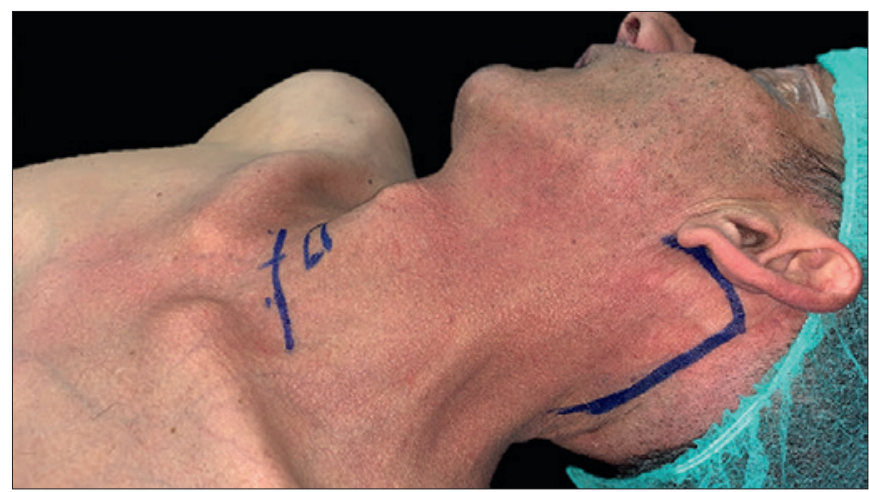

Figure 8. Modified retroauricular or facelift skin incision and cervical median skin window. 
median skin window. In this step the VITOM-3D allows to magnify the vision of the lower part of IV level, increasing the precision of the dissection and haemostatic manoeuvers near the inferior cervical pedicle, phrenic nerve and major lymphatic structures.

\section{Dissection of level $\mathrm{Ib}$}

For dissection of level Ib, the first surgeon must be positioned more caudally, directing surgical instruments cranially. The retractor blade must be positioned medially higher on the skin flap. The assistant also uses a skin hook to pull up the skin flap. The direction of the exoscope is oriented more cranially toward the upper part of the neck.

After dissecting the superficial cervical fascia, the submandibular gland is exposed, and the facial vein is identified on the surface of its posterior pole. The distal facial pedicle is carefully ligated $1 \mathrm{~cm}$ from the inferior margin, thus avoiding inadvertent damage to the mandibular branch of the VII ${ }^{\text {th }}$ cranial nerve; this manoeuver is made easier by the excellent 3D vision provided by the exoscope. Submandibular gland removal begins from the anterior pole. Considering the working distance and the presence of blood vessels on the muscular surfaces, it can be very useful to carry out this dissection using $24 \mathrm{~cm}$ LigaSure Maryland forceps, in haemostatic mode (Fig. 9).

Dissection of level $\mathrm{Ib}$ is carried out completely under 3D exoscopic vision and, in our experience, was particularly facilitated and precise.

\section{Discussion}

In 1905, Crile was the first to propose a systematic classification of neck dissection. Radical neck dissection has historically been the treatment of choice for the manage-

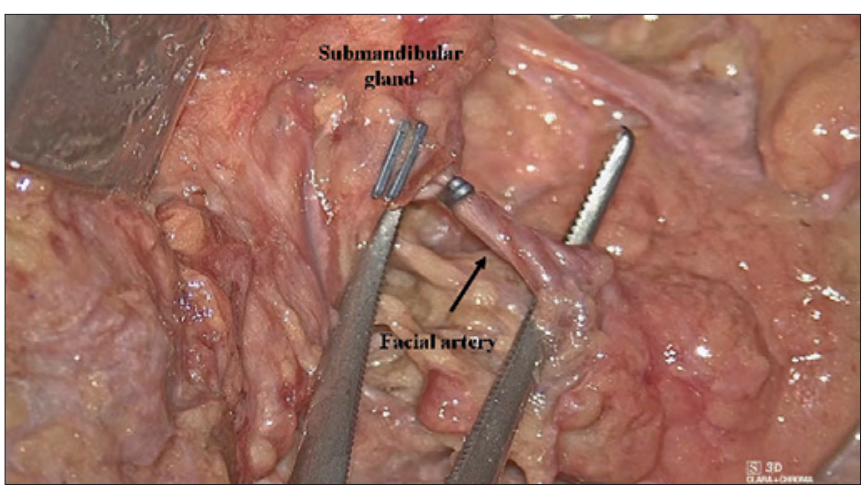

Figure 9. Surgical view of submandibular gland removal (right side): considering the working distance and the presence of blood vessels on the muscular surfaces, it can be very useful to carry out this dissection using a $24 \mathrm{~cm}$ LigaSure Maryland forceps, in haemostatic mode. ment of metastases of head and neck malignant tumours ${ }^{9}$. The surgical technique has changed over time with the introduction of more conservative approaches, curative and elective, allowing the preservation of anatomical structures such as the IJV, accessory spinal nerve, and sternocleidomastoid muscle ${ }^{10}$.

Carrying out a neck dissection using the traditional approach does not simply mean the presence of a more or less visible scar on the neck, but it can expose the patient to more easily acquired and more frequent complications such as cervical lymphoedema, fibrosis, scar retraction, keloids and wound dehiscence, that can have a negative impact on the perception of the body's self, especially in young and female patients ${ }^{11}$. In addition, since the neck dissection is performed in combination with transoral surgery in a good percentage of cases, this partly undermines the patient's perception of the concept of "mini-invasiveness" of the latter. All this configures the condition of carrying out a truly minimally invasive operation on primary carcinoma, with an even greater economic weight linked to technological devices, keeping the aesthetic and functional cervical sequelae unchanged due to the open neck approach by dissecting the lymph node areas. In any case, the aesthetic and functional outcomes after robotic surgery, widely validated in terms of minimal invasiveness and overall lower weight, justify the use of this device in the transoral treatment of some primary tumours coupled with a traditional open neck approach.

In recent years, head and neck oncologic surgery has been developing various forms of minimally invasive surgical procedures to increase surgical precision as well as the satisfaction and quality of life of patients. In 2010, Kang and colleagues first described the so-called RAND (remote access neck dissection), or robot-assisted neck dissection, for the treatment of cervical metastases from thyroid carcinoma using a transaxillary approach, similar to that used for total thyroidectomy ${ }^{12}$. Based on these observations, in 2012, Koh and colleagues proposed the modified retroauricular and facelift approaches for performing robotassisted neck dissection, not only of levels II, III, and IV but also I and V, highlighting the greater ease in dissecting upper and posterior cervical levels ${ }^{13}$. The main limitation highlighted using this access route was the possibility of performing the dissection of levels IIb and Va, given the limited vision of these regions.

Although these access routes cannot be literally defined as "minimally invasive", they are not burdened by the impact of the scar, which is virtually invisible and not a decisive factor in the main sequelae of the standard cervical approach; they can therefore be equated as outcomes with currently minimally invasive interventions, referring to them as "remote access" interventions. 
Concerning endoscopic-assisted neck dissection, several drawbacks have been highlighted by many studies ${ }^{14-17}$. These limitations include a reduced range of motion, with various collisions between first surgeon and assistant, a two-dimensional view with a lack of depth perception, impaired eye-hand coordination, minimal tactile sensation, and a relatively slow learning curve. Another important disadvantage of endoscopic neck dissection is the need for the operator to have sufficient skill in handling the endoscope. The focus of recent research has been shifting towards greater implementation of the da Vinci surgical robot, as this technique addresses some drawbacks of endoscopic surgery. The robotic surgical system has the advantage of a stable three-dimensional binocular magnification, motion scaling, tremor filtration, various degrees of freedom with wrist-like articulated movements, a shorter learning curve, superior surgeon ergonomics and improved instrumental dexterity. Although robotic surgery undoubtedly has clear advantages, comparative studies of endoscopic- and robotic-assisted techniques have not been able to illustrate differences in terms of outcomes from a simple comparison of numbers such as blood loss, conversion rate, or recovery time ${ }^{3,4,13,18}$.

The main disadvantages of robotic-assisted neck dissection are the length of the operation, significantly longer than the standard approach, the need for surgeons to develop skills in the field of robotic techniques and the higher overall costs of the procedure.

The recent advent of 3D exoscopic surgery has allowed interesting technical improvements to be introduced in head and neck surgery, with technical solutions applicable to cervical dissection, with the aim of enhancing surgical vision through remote access, minimising the costs of the procedure in comparison to robotic surgery.

Robotic surgery and VITOM-3D have only one characteristic in common: a high definition optical system (even though different in optical type and positioning with respect to the working area: in fact, one is a 3D-endoscopic device and its tip reaches near the anatomical structures, while the second is a 3D-endoscopic device placed outside the patient) allowing magnification and improved vision of anatomical sites otherwise difficult to explore without a direct conventional approach. Based on these considerations, we recently conducted this preclinical investigation in the cadaver lab focused on approaching conventional neck dissection using a retroauricular incision, and evaluate the applications and usefulness of the Storz 3D Exoscopic System during different stages of the surgical procedure. The acronym RAND-3D (3D exoscopic surgery) was coined to describe the use of this optical tool in neck dissection.

The current study in the cadaver lab indicates that RAND-
$3 \mathrm{D}$ is an acceptable alternative operating technique performing neck dissection by a retroauricular approach. Technically feasible, this technique assures a complete compartment-oriented dissection.

No particular difficulties were encountered during dissection of levels II and III; these steps are mainly conducted under direct vision with $3.5 \times$ magnification and with a small percentage (10-20\%) of 3D-assisted vision, which is particularly useful for the second surgeon. To facilitate a straightforward and rapid dissection of levels IV and V, the proposed solution is to combine the retroauricular incision with a small incision placed midway between the cricoid cartilage and the sternal notch, as is currently used in thyroid surgery. This solution is extremely useful in reducing the operating time and could be considered an interesting solution during robotic-assisted neck dissection in the case of major bleeding which is difficult to manage in the lower anatomic regions of the neck. Furthermore, it results in a small median lower neck skin incision, transforming a scar-free surgical procedure (as the purely RAND technique offers) into one with a minor scar, but provides the surgeons with great confidence in dissecting areas distant from cutaneous access and, if necessary, quickly convert to a retroauricular approach into a more comfortable procedure without paying the price of a major scar in the neck.

It should also be noted that these operations are sometimes associated with a tracheostomy to protect the airway: the same procedure is carried out at the end of the procedure by the same service incision. In the same way, in some borderline cases due to comorbidity, thanks to this incision, access to the trachea can be prepared without opening it. This creates a pre-tracheotomy situation, which can be easily opened to the patient's bed in the case of major bleeding complications in the postoperative period.

The hybrid execution of neck dissection under direct and exoscopic vision represents a valid alternative to video-assisted endoscopic- and robot-assisted techniques. Undoubtedly, the need for a second skin incision could underpower the good aesthetic results of the approach.

The characteristics of the VITOM-3D images can be considered very comparable to those of the 3D optics of the da Vinci surgical robot, with its excellent ability to provide $3 \mathrm{D}$ visual information which is used to interactively control the exoscope camera. Other advantages are its depth of field, magnification, and image contrast and colour, allowing direct manipulation of the images of anatomic structures. The most advantageous aspects are represented by the magnification of the anatomic details, for example, the vascularisation.

The system is comfortable to use for surgeons who stay for long periods in a sitting position with the screen in front of 
them at eye height: surgery carried out facing a 3D screen is not bothersome for operators, even for longer procedures. After carrying out the first procedure, the combination of direct vision and 3D exoscopic vision is very easy to work with. In fact, adapting and placing a polarised lens over the loupes allowed us to reach a comfortable optical solution. Almost without realising it, the surgeons pass from one vision to another depending on the best visual alternative. This continuous comparison between exoscope and loupes allowed us to reach some conclusions. VITOM-3D allows to share the same vision among surgeons, placed on the same side of the cadaver through a limited skin window, during the entire procedure. The exoscope is particularly useful to the second surgeon during haemostatic manoeuvers and to place multiple vascular clips in the dissection of the lower part of IV and V level. 3D visualisation is essential during level $\mathrm{Ib}$ dissection to better identify each anatomical structure, whereas it is almost impossible to easily dissect level Ia using this approach, due to the working distance.

Conventional surgical instruments have been used (no requirement to buy other specific instrumentation): it is only required that the surgical instruments are of suitable length (minimum $24 \mathrm{~cm}$ ). Other haemostatic tools can be used (e.g. Focus, LigaSure, Thunderbeat) with safety deriving from complete visual control. These aspects are undoubtedly an advantage, in terms of immediacy, simplicity of use and lower costs in comparison to robotic surgery.

Furthermore, the 3D exoscope provides the benefit of great utility in the learning process, especially for residents, fellows, students, and OR staff, thanks to the same shared visual experience being available to every operator, and always with wide high-resolution monitors. Both images and video sequences can be stored in high definition, enabling surgeons to share videos in didactic sessions, meetings and surgical technique courses. This utility applies not only to neck dissection, but also to the treatment of primary tumours ${ }^{8}$.

At present, in a health policy aimed at reducing costs, it is difficult to procure up-to-date technologies. The entire cost of the exoscopic platform is similar to that of an operating microscope with an electromagnetic brake. The cost of disposables for each surgical procedure is about €40-60, composed of two sterile sheaths for the holder and the controller chamber; even the cost of maintenance is considerably lower. Much of the same platform can be used daily in endoscopic surgery of the upper aero-digestive tract, the most frequently performed endoscopic procedure, and this contributes greatly to the amortisation of costs.

The current drawbacks are represented by the mechanical holder arm which is not always comfortable to move during surgery, and the necessity to wear 3D glasses for a prolonged period which can lead to headaches in some cases ${ }^{19-21}$. In our study, none of the surgeons complained of side effects.

Another important aspect to underline is that the RAND3D optical tool could be used in preparation for roboticassisted neck dissection, both for the fidelity of vision and for the logistics that make it more easily transportable in the cadaver lab than the robotic platform.

\section{Conclusions}

The primary endpoint of this study was to demonstrate the effectiveness and feasibility of this technology in retroauricular or facelift approaches to neck dissection.

The preclinical investigations on VITOM-3D-assisted neck dissection via a retroauricular approach (RAND-3D) have demonstrated good. This approach can be used in selected cases with a clear cosmetic benefit and representing a valid alternative to endoscopic- and robotic-assisted neck dissection. Nevertheless, we have concluded that, with the retroauricular approach, it is possible to perform a large part of the neck dissection under direct vision, even using a minimally invasive approach. The exoscope is useful because it allows the second operator, seated on the same side as the first surgeon, to have a good vision, especially during the dissection of levels II and III, improving precision during haemostasis. In our opinion, dissection of level IV exclusively through a retroauricular skin incision is quite unsafe using this technique because the working space is extremely narrow. This is the reason why we suggest a median skin "window" which permits better vision and greater safety in the dissection of vascular and lymphatic structures.

The preliminary results obtained in the cadaver lab have led us to propose to the Ethics Committee of our Institute a perspective study focused on the oncological and esthetic results obtained by RAND-3D with more specific evaluations of costs, patient satisfaction, and learning curve. That study is currently ongoing.

Moreover, we are planning an additional study to compare VITOM-3D and DaVinci systems in carrying out neck dissection with retroauricolar approach.

\section{References}

1 Weinstein GS, O’Malley BW Jr, Snyder W, et al. Transoral robotic surgery: radical tonsillectomy. Arch Otolaryngol Head Neck Surg 2007;133:1220-6. https://doi.org/10.1001/archotol.133.12.1220

2 Weinstein GS, O’Malley BW Jr, Snyder W, et al. Transoral robotic surgery: supraglottic partial laryngectomy. Ann Otol Rhinol Laryngol 2007;116:19-23. https://doi. org/10.1177/000348940711600104

3 Kim WS, Lee HS, Kang SM, et al. Feasibility of robot-assisted 
neck dissections via a transaxillary and retroauricular ("TARA") approach in head and neck cancer: preliminary results. Ann Surg Oncol 2012;19:1009-17. https://doi.org/10.1245/s10434-011-2116-2

4 Kim WS, Byeon HK, Park YM, et al. Therapeutic robot-assisted neck dissection via a retroauricular or modified facelift approach in head and neck cancer: a comparative study with conventional transcervical neck dissection. Head Neck 2015;37:249-54. https://doi.org/10.1002/ hed.23595

5 Lee HS, Kim WS, Hong HJ, et al. Robot-assisted supraomohyoid neck dissection via a modified face-lift or retroauricular approach in earlystage cN0 squamous cell carcinoma of the oral cavity: a comparative study with conventional technique. Ann Surg Oncol 2012;19:3871-8. https://doi.org/10.1245/s10434-012-2423-2

6 Lewis JS Jr, Carpenter DH, Thorstad WL, et al. Extracapsular extension is a poor predictor of disease recurrence in surgically treated oropharyngeal squamous cell carcinoma. Mod Pathol 2011;24:1413-20. https://doi.org/10.1038/modpathol.2011.105

7 Koh YW, Kim JW, Lee SW, et al. Endoscopic thyroidectomy via a unilateral axillo-breast approach without gas insufflation for unilateral benign thyroid lesions. Surg Endosc 2009;23:2053-60. https:// doi.org/10.1007/s00464-008-9963-3

8 Crosetti E, Arrigoni G, Manca A, et al. 3D exoscopic surgery (3Des) for transoral oropharyngectomy. Front Oncol 2020;10:16. https://doi. org/10.3389/fonc.2020.00016

9 Crile GW. On the surgical treatment of cancer of the head and neck. With a summary of one hundred and five patients. Trans South Surg Gynecol Assoc 1905;18:108-27.

10 Robbins KT, Clayman G, Levine PA, et al. Neck dissection classification update: revisions proposed by the American Head and Neck Society and the American Academy of Otolaryngology-Head and Neck Surgery. Arch Otolaryngol Head Neck Surg 2002;128:751-8. https:// doi.org/10.1001/archotol.128.7.751

11 Terrell JE, Nanavati KA, Esclamado RM, et al. Head and neck cancer-specific quality of life: instrument validation. Arch Otolaryngol Head Neck Surg 1997;123:1125-32. https://doi.org/10.1001/archotol.1997.01900100101014
12 Kang SW, Jeong JJ, Yun JS, et al. Robot-assisted endoscopic surgery for thyroid cancer: experience with the first 100 patients. Surg Endosc 2009;23:2399-406. https://doi.org/10.1007/s00464-009-0366-x

13 Koh YW, Chung WY, Hong HJ, et al. Robot-assisted selective neck dissection via modified face-lift approach for early oral tongue cancer: a video demonstration. Ann Surg Oncol 2012;19:1334-5. https:// doi.org/10.1245/s10434-011-2155-8

14 Kang SW, Jeong JJ, Yun JS, et al. Gasless endoscopic thyroidectomy using trans-axillary approach; surgical outcome of 581 patients. Endocr J 2009;56:361-9. https://doi.org/10.1507/endocrj.k08e-306

15 Lombardi CP, Raffaelli M, Princi P, et al. Minimally invasive videoassisted functional lateral neck dissection for metastatic papillary thyroid carcinoma. Am J Surg 2007;193:114-8. https://doi.org/10.1016/j. amjsurg.2006.02.024

16 Lira RB, Chulam TC, Koh YW, et al. Retroauricular endoscopeassisted approach to the neck: early experience in Latin America. Int Arch Otorhinolaryngol 2016;20:138-44. https://doi. org/10.1055/s-0036-1578807

17 Lee S, Nam W. Comparison of retroauricular and small transcervical approaches for endoscopic-assisted selective neck dissection: a cadaveric study. Int J Oral Maxillofac Surg 2019;48:584-89. https://doi. org/10.1016/j.ijom.2018.11.008

18 Kim CH, Chang JW, Choi EC, et al. Robotically assisted selective neck dissection in parotid gland cancer: preliminary report. Laryngoscope 2013;123:646-50. https://doi.org/10.1002/lary.23716

19 Garneau JC, Laitman BM, Cosetti MK, et al. The use of the exoscope in lateral skull base surgery: advantages and limitations. Otol Neurotol 2019;40:236-40. https://doi.org/10.1097/MAO.0000000000002095

20 Chen J, Zheng H, He J. 3D laparoscopic revision thyroidectomy through an anterior chest approach: a case report. J Laparoendosc Adv Surg Tech A 2015;25:238-42. https://doi.org/10.1089/lap.2014.065

21 Capaccio P, Di Pasquale D, Bresciani L, et al. 3D video-assisted transoral removal of deep hilo-parenchymal sub-mandibular stones. Acta Otorhinolaryngol Ital 2019;39:367-73. https://doi.org/10.14639/0392$100 \mathrm{X}-2282$ 